\title{
4-dimensional spectral-tomography of carbonaceous
}

\section{nano-composites}

\author{
Mhairi H. Gass* and Paul A. Midgley \\ Department of Materials Science and Metallurgy, University of Cambridge, Pembroke Street, \\ Cambridge, CB2 3QZ, UK
}

Corresponding author. E-mail: mhg25@cam.ac.uk

\section{The projection requirement}

To be suitable for tomographic reconstruction the transmitted signal must meet the projection requirement; the projected signal must be a monotonically varying function of thickness. ${ }^{1,2}$ For crystalline objects, diffraction contrast dominates the BF images. Diffraction contrast is highly dependant on crystal orientation, has no monotonic relationship with thickness, and therefore does not meet the projection requirement. The contrast observed in energy-loss images is a combination of inelastic and elastic scattering. By dividing two energy-filtered windows (e.g. for the conventional jump-ratio elemental analysis) residual diffraction contrast is removed helping to fulfill the projection requirement. The jump-ratio increases with increasing thickness up to a maximum value at a critical thickness $t_{\text {crit }}$, and then starts to fall because of the decreasing signal-to-background ratio. Thicker regions may produce lower jump-ratios than thinner areas, invalidating the projection requirement. It is important that the specimen does not exceed the thickness $t_{\text {crit }}$ at any tilt. It is generally accepted that the 
jump-ratio signal changes monotonically with thickness up to $\sim 1-1.5 \times$ the inelastic mean free path, $\lambda$. The inelastic mean free path can be approximated from ${ }^{3}$

$$
\lambda \approx \frac{106 F\left(E_{0} / E_{m}\right)}{\ln \left(2 \beta E_{0} / E_{m}\right)},
$$

where

$$
F=\frac{1+E_{0} / 1022}{\left(1+E_{0} / 511\right)^{2}}
$$

is a relativistic factor, the incident beam energy $E_{0}$ is in $\mathrm{keV}, \beta$ the objective semi-angle in mrad and $E_{m}$ represents the average energy-loss in $\mathrm{eV}$. Values of $\mathrm{E}_{\mathrm{m}}$ for common materials may be found in the literature. ${ }^{3}$ For carbon, values of $\mathrm{E}_{\mathrm{m}}$ are in the range of $15 \mathrm{eV}$. For a microscope with an incident beam energy of $300 \mathrm{keV}$ and $\beta$ of $10 \mathrm{mrad}$, the inelastic mean free path is $\sim 180 \mathrm{~nm}$. The nylon / nanotube specimen is estimated to be $100 \mathrm{~nm}$ at its thickest, considerably thinner than the mean free path and thus meeting the projection requirement.

\section{Low-loss spectra of carbonaceous materials}

Although energy-filtered TEM (EFTEM) allows for the fast acquisition of high spatial resolution elemental maps ${ }^{2,4}$ for carbonaceous composites conventional core-loss EFTEM mapping is of limited value. The fine structure that is seen at the onset of the carbon ionization edge in energy-loss spectroscopy $^{5}$ offers a means to map varying carbon species but in practice many polymeric matrices or binders will damage with the high electron dose needed for this type of mapping. Other techniques,

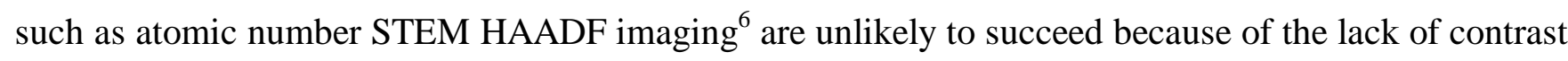
between the composite components of interest. Therefore an alternative, relatively low-dose, technique is needed that can distinguish between the carbonaceous components in the composite and one that also 
satisfies the projection requirement to enable tomographic reconstruction and subsequent 3dimensional analysis. ${ }^{3}$

The low-loss region of the energy-loss spectrum ( 0 to $50 \mathrm{eV})$ has two advantages for imaging carbonaceous species: (i) the scattering cross-sections are many orders of magnitude larger than for core-loss scattering, and thus acquisition times and beam damage can be kept to a minimum, and (ii) the $(\pi+\sigma)$ volume plasmon excitation which dominates the low-loss spectrum is sensitive to the electronic structure of the material studied. One other transition has been indicated on the spectrum from the nanotube and nylon at $6 \mathrm{eV}$, the $\pi \rightarrow \pi^{*}$ transition, resulting from the fourth valence electron in the graphitic nanotubes which forms the $\pi$ bond, absent in the nylon. The $\pi \rightarrow \pi^{*}$ transition is orientation-dependent and as a specimen is tilted away from the c-axis, the relative intensity of the $\pi \rightarrow \pi^{*}$ transition decreases; ${ }^{7}$ it is therefore unsuitable as a signal for tomographic reconstructions. The spatial resolution of the plasmon image is dominated by the chromatic aberration of the microscope objective lens and the spatial delocalization of the excited plasmon. However, using small energy windows ( $3 \mathrm{eV}$ in this case) at low energy-losses ensures the effects of chromatic aberration are minimized. Theoretical calculations show the resolution of a bulk plasmon to be limited by the delocalization and is estimated to be $\sim 1 \mathrm{~nm}$ at the plasmon energy.

\section{Pre-processing of plasmon images}

Before the ratio image from the two plasmon energies can be calculated the background signal from the vacuum must be set to a small non-zero constant value; the constants were set at 0.01 for the $22 \mathrm{eV}$ images and 1 for the $28 \mathrm{eV}$ images for the $22 \mathrm{eV} / 28 \mathrm{eV}$ ratio and 1 for the $22 \mathrm{eV}$ images and 0.01 for the $28 \mathrm{eV}$ images for the $28 \mathrm{eV} / 22 \mathrm{eV}$ ratios, this insures that the signal from the vacuum does not 'contaminate' the nano-composite reconstruction. 
Figure S1 shows low-loss image-spectra ${ }^{10}$ taken from nylon (blue) and from the MWCNT with a nylon coating (black). The main feature in both spectra is the bulk $(\pi+\sigma)$ plasmon, with a maximum at $22 \mathrm{eV}$ for the nylon and at $26 \mathrm{eV}$ for the nanotube-plus-nylon. It can be seen that the peak position of the plasmon for the nanotube is slightly lower than that generally quoted $(27 \mathrm{eV})$ and this is likely to be due to the presence of the nylon in the analyzed volume, resulting in an apparent shift to lower energies.

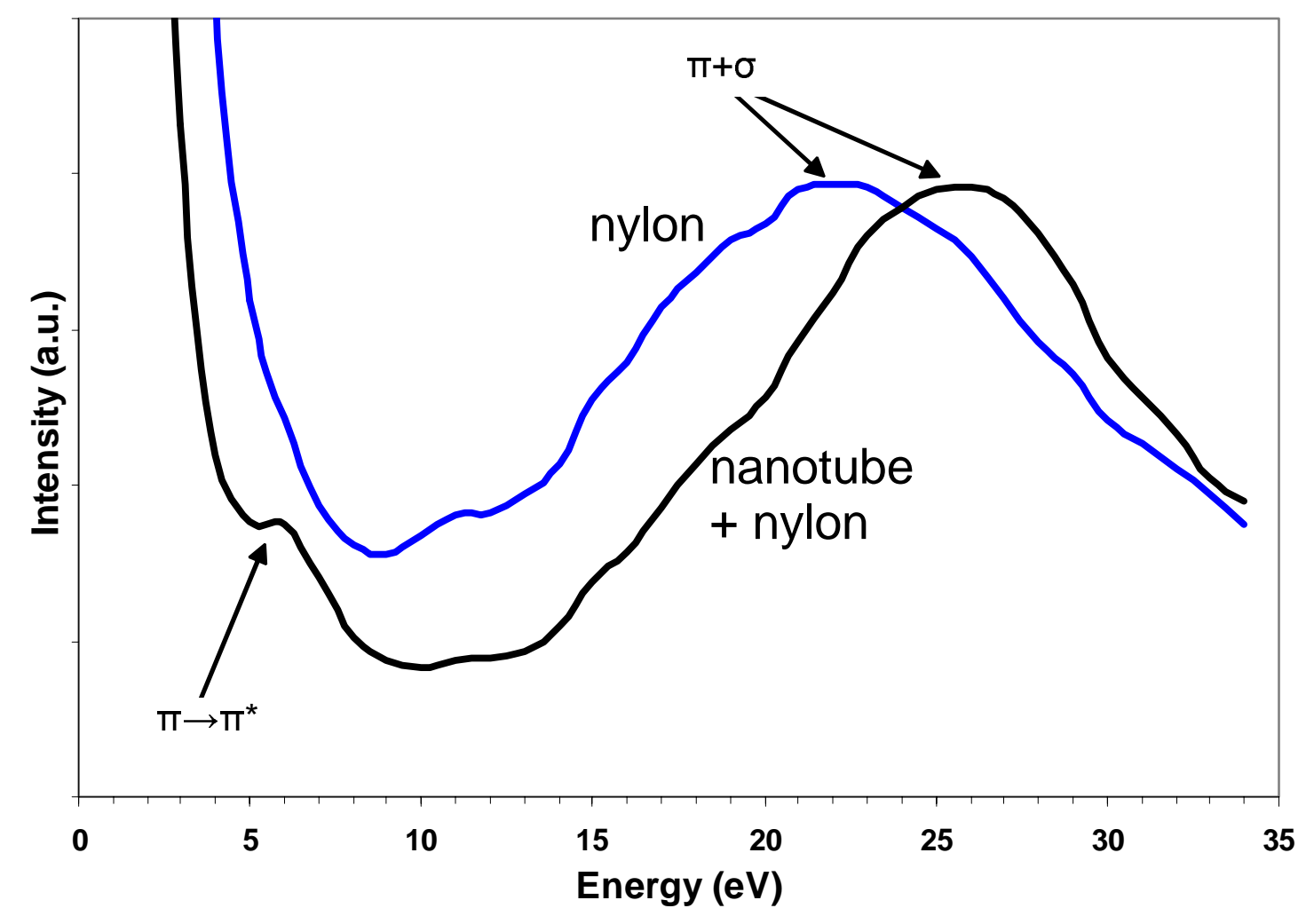

Figure S1. Low-loss energy-loss spectra for nylon (blue) and carbon nanotube coated with nylon (black) show the difference in the $\pi+\sigma$ plasmon energy. The presence of the $\pi \rightarrow \pi^{*}$ transition from the graphitic nanotube is also observed 


\section{References}

(1) Hawkes, P.W. The electron microscope as a structure projector In Electron Tomography: Three

dimensional imaging with the transmission electron microscope, 1992, J. Frank (Ed.) Plenum Press, New York, London

(2) Weyland, M.; Midgley, P.A. Microsc. Microanal., 2003, 9, 542

(3) Egerton, R.F. Electron Energy Loss Spectroscopy in the Electron Microscope, 1996, $2^{\text {nd }}$ Edition, Plenum Press, New York

(4) Midgley, P.A.; Weyland, M. Ultramicroscopy, 2003, 96, 413

(5) Papworth, A.J.; Kiely, C.J.; Burden, A.P.; Silva, S.R.P.; Amaratunga, G.A.J. Phys. Rev. B, 2000, 62,12628

(6) Midgley, P.A.; Weyland, M.; Thomas, J.M.; Johnson, B.F.G. Chem. Commun., 2001, 10, 907

(7) Daniels, H.R.; Brydson, R.; Brown, A.; Rand, B. Ultramicroscopy, 2003, 96, 547

(8) Egerton, R.F. J. Electron Microsc., 1999, 48, 711

(9) Grogger, W.; Schaffer, B.; Krishnan, K.M.; Hofer, F. Ultramicroscopy, 2003, 96, 481

(10) Thomas, P.J.; Midgley, P.A. Topics in Catalysis, 2002, 21, 109 